\title{
Anticipatory Suppression of Nonattended Locations in Visual Cortex Marks Target Location and Predicts Perception
}

\author{
Chad M. Sylvester, ${ }^{1}$ Anthony I. Jack, ${ }^{4}$ Maurizio Corbetta, ${ }^{1,2,3}$ and Gordon L. Shulman ${ }^{2}$ \\ Departments of ${ }^{1}$ Radiology, ${ }^{2}$ Neurology, and ${ }^{3}$ Anatomy and Neurobiology, Washington University School of Medicine, St. Louis, Missouri 63110, and \\ ${ }^{4}$ Department of Cognitive Science, Case Western Reserve University, Cleveland, Ohio 44016
}

\begin{abstract}
Spatial attention is associated with modulations in prestimulus, anticipatory blood oxygen level-dependent (BOLD) activity across the brain. It is unclear, however, if these anticipatory modulations depend on the computational demands of the upcoming task. Here, we show that anticipation of low-contrast stimuli, relative to high-contrast stimuli, is associated with increased prestimulus BOLD activity in the frontal eye field (FEF) and the posterior inferior frontal sulcus (IFS) but not in the intraparietal sulcus (IPS). In visual cortex, anticipation of low-contrast stimuli is associated with increased suppression of activity corresponding to unattended (but not attended) locations, and this suppression predicts whether subjects will accurately perceive low-contrast stimuli. These results suggest that when a stimulus will be difficult to distinguish from the background, top-down signals from FEF and IFS can facilitate perception by marking its location through the suppression of unattended locations in visual cortex.
\end{abstract}

Key words: attention; contrast; fMRI; suppression; topography; visual

\section{Introduction}

Under natural conditions, the perception of behaviorally relevant objects may be limited by different perceptual factors. At one extreme, an object may be barely visible against the surrounding background, such as a car on a road immersed in a thick fog. At the other extreme, an object may be highly visible but difficult to discriminate from a similar object, as when trying to distinguish two cars from successive model years based on the shape of their grilles.

A fundamental question is whether and how the computational demands imposed by a task affect the attentional mechanisms involved in task performance (Eriksen and Hoffman, 1974; Posner et al., 1980; Carrasco et al., 2000; Dosher and Lu, 2000; Carrasco, 2006). For example, attention could aid perception in both threshold detection and suprathreshold discrimination (the two situations described above) by boosting the signal related to the task-relevant object, so-called "signal enhancement." Only the threshold detection task, however, would benefit from excluding noise from nearby locations, so-called "noise exclusion," because noise from nearby empty locations would not be confused for the target in the suprathreshold discrimination task (Carrasco et al., 2000; Dosher and Lu, 2000).

Received Jan. 21, 2008; revised April 21, 2008; accepted May 18, 2008.

This work was supported by the J. S. McDonnell Foundation, National Institute of Neurological Disorders and Stroke Grants F30 NS057926-01 and R01 NS48013, National Institute of Mental Health Grant R01 MH71920-06, and Marie Curie Chair European Union Grant MEXC-CT-2004-006783. We thank Drs. Giovanni d'Avossa, Ayelet Sapir, and Mark McAvoy for guidance with statistical analyses.

Correspondence should be addressed to Chad M. Sylvester, Washington University School of Medicine, $4525 \mathrm{Scott}$ Avenue, Department of Radiology Campus Box 8225, St. Louis, M0 63110. E-mail: chad@npg.wustl.edu. D01:10.1523/JNEUROSCI.0275-08.2008

Copyright $\odot 2008$ Society for Neuroscience $\quad$ 0270-6474/08/286549-08\$15.00/0
Despite the fact that attention could improve threshold detection and suprathreshold discrimination in part through different mechanisms, it is unclear whether and how the neural signals implementing spatial attention depend on task demands. Prestimulus, preparatory activations in higher-order frontal and parietal regions are thought to implement the top-down control of spatial attention, in part by modulating prestimulus activity in the visual cortex (Hopfinger et al., 2000; Kastner and Ungerleider, 2000; Corbetta and Shulman, 2002). In support, threshold detection tasks are associated with anticipatory activation in the retinotopic portion of visual cortex corresponding to the attended location (Jack et al., 2006; Silver et al., 2007; Sylvester et al., 2007) along with deactivations in corresponding nontarget locations (Silver et al., 2007; Sylvester et al., 2007). Similar prestimulus activations (Kastner et al., 1999; Muller et al., 2003; Serences et al., 2004; McMains et al., 2007) and deactivations (Muller and Kleinschmidt, 2004) have been reported in suprathreshold discrimination tasks. It is unknown, however, whether the magnitude and spatial distribution of these prestimulus signals in both retinotopic visual cortex and higher-order regions are related to the different perceptual demands imposed by the different tasks.

Here, we explicitly test how the neural implementation of spatial attention varies with task demands by comparing prestimulus blood oxygen level-dependent (BOLD) activity on a threshold detection task versus a fine suprathreshold discrimination task. Critically, the tasks are matched for difficulty, effects of attention on performance, and the spatial distribution of stimuli. The most straightforward prediction is that deactivations corresponding to unattended locations are stronger in the threshold detection task, because this task could benefit from noise exclu- 
sion, whereas the activations corresponding to the attended location are stronger in the suprathreshold discrimination task, because this task may rely solely on signal enhancement.

\section{Materials and Methods}

Subjects. Six subjects (three female) were recruited, aged 26-30, righthanded, with no history of neurological illness, and normal or correctedto-normal vision. Informed consent was obtained as per the guidelines of the human studies committee at Washington University School of Medicine. Subject 2 was author CS.

Task. Eye position was monitored to ensure subjects always fixated a central crosshair. Each trial began with a $500 \mathrm{~ms}$ auditory "preparatory" cue, the spoken word "left" or "right," indicating one of two locations: $5^{\circ}$ eccentricity and $45^{\circ}$ of radial angle to the left or right of the vertical meridian (see Fig. 1). After a stimulus-onset asynchrony (SOA) of 6.192 s (25\%), $8.256 \mathrm{~s}(25 \%)$, or $10.32 \mathrm{~s}(50 \%)$, targets appeared for $100 \mathrm{~ms}$ centered at both locations, concurrent with an auditory report cue ("left" or "right"). Targets were 3.5 cycle-per-degree Gabor patches with a Gaussian envelope SD of $0.3^{\circ}$. On valid trials (75\%), the report cue matched the preparatory cue. Subjects indicated the orientation (left tilt, vertical, right tilt) of the report-cued Gabor with a button press. Subjects could indicate their response at any time after the presentation of the target stimulus and before the beginning of the next trial. There was a random intertrial interval (ITI) of $16.512 \mathrm{~s}$ (33\%), $18.576 \mathrm{~s}(33 \%)$, or $20.64 \mathrm{~s}(33 \%)$. Each $6.2 \mathrm{~min}$ functional magnetic resonance imaging scan block consisted of 13 trials; each subject performed between 1450 and 1750 trials over 8-12 scanning sessions. Subject 5 performed only 1144 trials. Scanning blocks with "high-contrast" and "low-contrast" targets (see below, Practice sessions and target parameters) were intermixed. Subjects were always aware of block type.

Practice sessions and target parameters. Before test scans, each subject performed $\sim 600$ high-contrast and 600 low-contrast practice trials, in two in-scanner sessions. The purpose of these sessions was to determine stimulus parameters that would plateau performance at $70 \%$ for each contrast. Timing was $2 \mathrm{~s}$ SOA and 2-4 s ITI. Across the six subjects, for the low-contrast stimuli, contrast at plateau performance ranged from 5 to $12 \%$, and the difference in orientation between targets varied from 6 to $45^{\circ}$. For high-contrast trials, contrast was maintained at $50 \%$, and the difference in orientation between targets at plateau performance ranged from 1.5 to $3.0^{\circ}$. Occasional small adjustments were also made during test sessions.

Data acquisition. Images were acquired with a Siemens Allegra 3T scanner. Structural images used a sagittal magnetization-prepared rapidacquisition gradient echo T1-weighted sequence [repetition time (TR), $1810 \mathrm{~ms}$; echo time (TE), $3.93 \mathrm{~ms}$; flip angle, $12^{\circ}$; inversion time, 1200 $\mathrm{ms}$; voxel size, $1 \times 1 \times 1.25 \mathrm{~mm})$. Blood oxygenation level-dependent (BOLD) contrast images were acquired with an asymmetric spin-echo echoplanar sequence (TR, $2.064 \mathrm{~s}$; TE, $25 \mathrm{~ms}$; flip angle, $90^{\circ}$; 32 contiguous $4 \mathrm{~mm}$ axial slices; $4 \times 4 \mathrm{~mm}$ in-plane resolution). BOLD images were motion corrected within and between runs, and timing differences across slices were corrected. Images were resampled into $3 \mathrm{~mm}$ isotropic voxels and warped into a standardized atlas space (Talairach and Tournoux, 1988).

Apparatus. Stimuli were presented with a Power Macintosh G4 computer (Apple) using Matlab software (Mathworks) with the psychophysics toolbox (Brainard, 1997; Pelli, 1997). Images were projected to the head of the bore of the scanner via an LCD projector (Sharp LCD C20X) and viewed with a mirror attached to the head coil. A magnet-compatible fiber-optic key-press device recorded subject responses.

Monitoring of eye position. Eye position was measured in five of six subjects (not subject 2, author CS) with an ISCAN ETL-200 system as subjects performed the task in the scanner. Author CS constantly viewed live video displaying subjects' eyes and any session in which a gross eye movement was made was immediately stopped and the data discarded. This occurred in fewer than five instances.

Linear modeling. The BOLD data at each voxel, for each subject, were subjected to a general linear model using in-house software. Constant and linear terms over each BOLD run accounted for baseline and linear drift, and sine waves modeled low-frequency noise $(<0.009 \mathrm{~Hz})$. Separate $\delta$ function regressors coded each of the 11-13 time points (22.0704$26.832 \mathrm{~s}$, depending on the SOA) after the preparatory cue of each of the 48 different event types [3 SOAs $\times$ (high vs low contrast) $\times$ (left vs right cue $) \times($ valid vs invalid $) \times($ correct vs incorrect $)]$. A "residuals" dataset was created by summing the modeled responses with the residuals unaccounted for by the linear model.

Derivation of regions of interest. In all analyses, only trials with the longest SOA [five magnetic resonance (MR) frames] were used, to avoid contaminating preparatory signals with stimulus-evoked activity. To create regions of interest (ROIs) outside of retinotopic cortex, we performed a voxelwise ANOVA over the first six trial time points using the residuals dataset, separately in each subject. The ANOVA effects of interest were cue direction, target contrast, validity, performance, and time. An inhouse clustering algorithm defined ROIs based on the resulting map of the main effect of time. ROIs started as $8 \mathrm{~mm}$ spheres centered on map peaks with $z$-scores $>10$; spheres within $12 \mathrm{~mm}$ of each other were consolidated into a single ROI. ROIs were then masked with the main effect of time image at a threshold of 3. An ROI was retained for subsequent analyses if present in at least 10 of 12 subject hemispheres. This procedure yielded ROIs (see Fig. 2) in the cingulate sulcus (CING), the superior frontal gyrus (SFG), the frontal eye field (FEF), the middle frontal gyrus (MFG), the inferior frontal sulcus (IFS), the anterior intraparietal sulcus (aIPS), the posterior intraparietal sulcus (pIPS), and the superior temporal gyrus (STG). We verified that each region in each subject occupied a single cortical location on a flattened representation of the subject's own anatomy using Caret software (Van Essen et al., 2001).

Additional ROIs were created within early visual cortex (V1-V4 and $\mathrm{V} 3 \mathrm{~A}$ ) representing different locations in the visual field. In a separate set of scans, subjects passively viewed high-contrast $(\sim 50 \%)$ Gabor patches flickering at $4 \mathrm{~Hz}$ in 12 s blocks. In each block, a Gabor randomly appeared at one of five locations: one of the two target locations, one of the two target locations mirrored across the horizontal meridian, and a single central location. We constructed contrasts of each passive stimulus with its mirror stimulus across the vertical meridian. The central location $\left(1^{\circ}\right.$ width) was contrasted with the summed responses to all other locations. In addition, subjects underwent retinotopy scans in which they passively viewed contrast reversing checkerboard stimuli extending along the horizontal and vertical meridians. A contrast of responses to the horizontal and vertical meridians was used to hand-draw borders of early visual regions on a flattened representation of the subject's own anatomy using Caret software (Van Essen et al., 2001). Subdivisions of early visual cortex were made by taking the conjunction of the voxels with a stimulus preference during the localizer scans $(z>2)$ and the earliest of the retinotopic regions (V1v, V2v, VP, and V4 for upper field locations; V1d, V2d, V3, and $\mathrm{V} 3 \mathrm{a}$ for lower field locations).

Time courses and statistical tests. In each ROI, data were averaged across voxels using the residuals dataset. Preparatory time courses (the first six time points from trials with the longest SOA) were extracted for each trial. For time course illustration, data were averaged across all trials of a particular type. Average responses were not rescaled to constrain BOLD activity to be 0 at cue onset. Group-wise statistics were regional three-way ANOVAs in which subject was treated as a random effect and the dependent variable was the preparatory BOLD activity before stimulus onset. For the initial analyses, the ANOVA effects of interest were cued location (contralateral vs ipsilateral), contrast (low vs high), and time (time points 1-6). In this analysis, data were combined across hemisphere. For the behavioral analyses, effects of interest were performance and time. Individual subject statistics were regional ANOVAs in which each trial was treated as a separate observation. Otherwise, methods were the same as for the group.

\section{Results \\ Behavior}

Subjects performed two versions of an orientation discrimination task that were matched for difficulty and for effects of spatial attention on performance. The task is depicted in Figure $1 A$. In low-contrast blocks, subjects discriminated the orientation of 

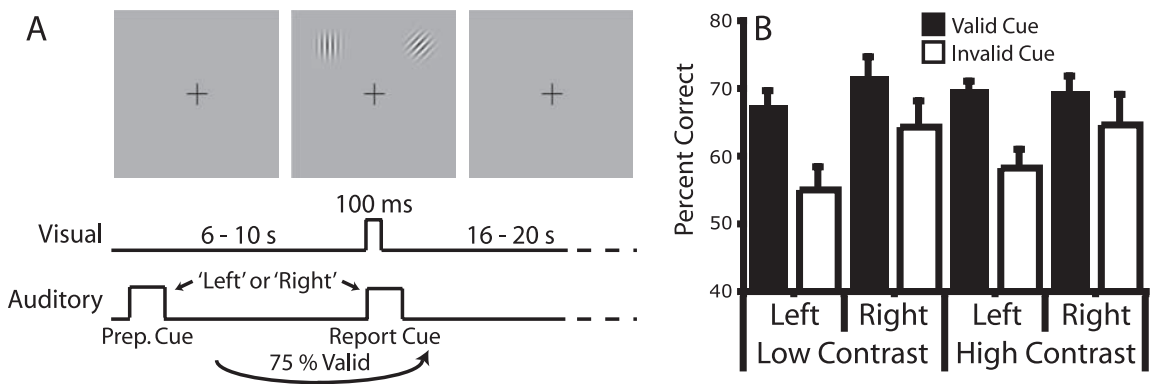

Figure 1. A, Task structure. Each trial began with an auditory preparatory cue indicating which of the two peripheral locations subjects should covertly attend. After a variable SOA, Gabor patches appeared briefly at both locations, coincident with an auditory cue indicating the target stimulus. Subjects reported the orientation of the Gabor at the target location with a button press. In some task blocks, targets were very low-contrast and performance was limited by visibility. In other blocks, targets were high-contrast and performance was limited by the small difference in orientation between target types. This study focused on BOLD activity after the preparatory cue but before the onset of the visual targets. $\boldsymbol{B}$, Subjects performed better after valid versus invalid preparatory cues, indicating that subjects attended to the location given by the preparatory cue. Behavior is matched across target contrast. Error bars indicate SEM.

Gabor patches that had very different orientations but were near contrast threshold so that perception was limited by the ability to detect the patches against the background. In high-contrast blocks, subjects discriminated the orientation of Gabor patches that had very similar orientations but were well above contrast threshold, so that perception was limited by the small difference in orientations between potential targets. Therefore, task structure and spatial distribution of stimuli were identical in the two conditions, although the perceptual demands were quite different.

Trials in both low and high-contrast blocks required carefully focused spatial attention throughout the interval between the auditory cue and the visual target onset. A four-way ANOVA (cue validity, target location, target contrast, and duration of cue-target interval) indicated that accuracy was significantly modulated by cue validity $\left(F_{(1,5)}=17.0 ; p=0.009\right)$, demonstrating that subjects directed attention to the cued location. Importantly, there was no effect of target contrast $\left[F_{(1,5)}=0.1 ; p\right.$ is not significant (n.s.) $]$ nor was there an interaction between cue validity and contrast $\left(F_{(1,5)}=0.2 ; p\right.$ is n.s. $)$, indicating that spatial attention was equally beneficial in low and high-contrast blocks. Furthermore, there was no effect of the duration of the cue-target interval $\left(F_{(2,4)}=3.6 ; p\right.$ is n.s. $)$, demonstrating that subjects sustained attention to the cued location throughout the interval.

A main effect of target location was observed $\left(F_{(1,5)}=6.3 ; p=\right.$ $0.053)$, because of slightly higher accuracy for targets presented in the right $(69.0 \%)$ than left visual field $(65.8 \%)$. There was also a significant interaction between target location and cue validity $\left(F_{(1,5)}=10.6 ; p=0.022\right)$, because the difference in accuracy between validly and invalidly cued trials was larger for left visual field targets $(11.8 \%)$ than for right visual field targets $(6.7 \%)$. These two latter findings indicate a slightly lower baseline accuracy in the left visual field, as well as a larger effect of attention on performance with left field targets. Task performance across different conditions is plotted in Figure $1 B$ (chance, 33\%).

These results show that differences in the spatial distribution of activations or deactivations in low and high-contrast conditions cannot be attributed to task difficulty or to the need for spatial attention, because these factors were matched across conditions.

\section{Preparatory activity in higher-order parietal and frontal regions}

First, we examined whether directing spatial attention while preparing to discriminate the orientation of low- versus highcontrast objects produced differential preparatory (prestimulus) responses in higher-order frontal and parietal regions that are the putative sources of attentional modulations in the visual cortex. Outside of the visual cortex, a significant response after the auditory spatial cue was observed in the FEF, SFG, CING, MFG, IFS, aIPS, pIPS, and STG. Critically, all of the following analyses of these regions concern only prestimulus, preparatory cue activity, which was likely related to several processes including processing of the auditory cue, directing spatial attention, and motor preparation. An example of a complete cue and target period time course is displayed in supplemental Figure 1 (available at www.jneurosci.org as supplemental material).

We next determined whether preparatory prestimulus activity in each of these regions depended on the cued location and the contrast of the upcoming target, as assessed by three-way regional ANOVAs [cue direction, upcoming target contrast, time (six time points)] on the time course of the BOLD response. Figure 2 shows time courses of preparatory prestimulus responses from frontal, parietal, and temporal regions active during the cue period, whereas Table 1 lists significant effects for each region. Preparatory activity in several regions was significantly higher when attention was directed into the contralateral hemifield (Fig. 2, compare green, orange curves): SFG $\left(F_{(1,5)}=10.32 ; p=0.024\right)$, $\operatorname{FEF}\left(F_{(1,5)}=76.03 ; p<0.001\right), \operatorname{IFS}\left(F_{(1,5)}=17.61 ; p<0.009\right)$, and pIPS $\left(F_{(1,4)}=13.34 ; p=0.022\right)$. However, only preparatory activity in $\operatorname{FEF}\left(F_{(1,5)}=6.10 ; p=0.057\right)$ and $\operatorname{IFS}\left(F_{(1,5)}=9.42\right.$; $p=0.028)$ was significantly modulated by the contrast of the upcoming target, with higher signals when the subsequent target was low contrast (Fig. 2, compare solid, dashed curves). Neither FEF nor IFS displayed a significant interaction between cue direction and contrast, indicating that the neural signals associated with the location of attention were independent from the signals associated with preparing for a particular contrast. Finally, some regions like STG, which was involved in auditory processing, were active during the cue period, but were modulated by neither cue direction nor contrast.

These results show that preparatory signals in FEF and IFS reflected both the location of attention and the upcoming task, whereas signals in IPS reflected only the location of attention.

\section{Preparatory activity in the visual cortex}

We next examined whether preparatory (prestimulus) activity across retinotopic visual cortex depended on whether subjects prepared to discriminate a low- or high-contrast object. Covert attention to a peripheral location was associated with increases in preparatory activity in the portion of visual cortex corresponding to the attended location and decreases in portions of visual cortex corresponding to unattended locations. For example, attending to the left upper peripheral visual field location (Fig. $1 A$, stimulus display) increased activity (relative to the intertrial interval) in portions of right ventral visual cortex (Fig. $3 A$ ) corresponding to the target location as determined by independent localizer scans 
(black outlines). Conversely, widespread decreases in activity were observed in nontarget locations, especially bilaterally in foveal and parafoveal locations (Fig. 3, eccentricity lines). Similar modulations were observed for rightward cues (Fig. 3).

To examine how these spatially selective modulations across the visual cortex depended on the task, we compared preparatory activity during low-contrast and highcontrast blocks for trials with leftward (Fig. $3 C$ ) or rightward attention (Fig. 3D). Preparatory activity in portions of visual cortex corresponding to unattended locations (bilateral fovea, lower visual field) was lower during low-contrast blocks relative to high-contrast blocks, whereas preparatory activity corresponding to the attended location was the same regardless of block type. This pattern was very similar after both leftward and rightward cues. Interestingly, the portion of cortex immediately adjacent to the representation of the target location displayed the largest suppression in low-contrast blocks relative to highcontrast blocks (see light blue regions near attended target locations between $3^{\circ}$ and $1.5^{\circ}$ degrees eccentricity iso-contours).

This qualitative impression was confirmed by a series of three-way regional ANOVAs [cued location, upcoming target contrast, time (six time points)] on the time course of the BOLD response (Fig. 4) in different parts of visual cortex as determined by independent localizers. Preparatory activity in regions representing locations that never contained a target and therefore were always unattended (i.e., lower visual field and central visual field) (Fig. 4) was significantly lower in lowcontrast than high-contrast blocks (main effect of target contrast, lower field, $F_{(1,5)}=$ $6.53, p=0.051$; central field, $F_{(1,5)}=35.16$, $p=0.002)$. The effect of contrast on preparatory activity in regions representing the two target locations depended on whether the target location was attended or unattended, as indicated by a significant interaction between contrast and cued location $\left(\mathrm{V} 1-\mathrm{V} 4, F_{(1,5)}=11.80, p=\right.$ 0.019 ; V3A, $\left.F_{(1,5)}=7.27, p=0.043\right)$. When the location was unattended, preparatory activity was lower in low-contrast than high-contrast blocks, but when the location was attended, preparatory activity was identical in the two blocks (Fig. 4). As expected, preparatory activity in retinotopic regions representing the target location was higher when that location was attended than when it was unattended (main effect of cued location, V1V4, $\left.F_{(1,5)}=12.09, p=0.018 ; \mathrm{V} 3 \mathrm{~A}, F_{(1,5)}=9.93, p=0.025\right)$. Significant modulations for each region are listed in Table 1.

In summary, retinotopic visual cortex showed (1) preparatory increases at attended target locations and decreases at unattended locations, (2) less activity at unattended locations when subjects expected a low than high-contrast object, and (3) similar preparatory activity at the attended location when subjects expected a low- or high-contrast object. This pattern suggests that noise is additionally suppressed at unattended locations when the upcoming target is difficult to detect.

\section{Correlation between neural activity and behavioral performance}

If preparatory suppression of activity at unattended locations aids detection of a subsequent low-contrast target, then greater suppression might be observed when the orientation of the target is accurately judged. We tested this hypothesis with respect to the preparatory activity in the central visual field, which showed the largest decrease in activity from high- to low-contrast blocks. Time courses are displayed in Figure 5. A two-way ANOVA [performance, time (six time points)] indicated that during lowcontrast blocks, activity representing the central visual field was significantly lower on correct than incorrect trials with leftward targets $\left(F_{(1,5)}=12.1 ; p=0.018\right)$. Preparatory activity did not predict performance for trials with rightward targets, but as noted earlier, the effect of attention on accuracy was also signifi- 
Table 1. Regions active following covert deployment of attention are modulated by the spatial locus of attention as well as whether subjects expect upcoming visual stimuli to be low or high contrast

\begin{tabular}{|c|c|c|c|}
\hline & \multicolumn{3}{|c|}{ Preparatory activity modulated by: } \\
\hline & Contrast & Cue direction & Contrast $\times$ cue direction \\
\hline \multirow{2}{*}{\multicolumn{4}{|c|}{$\begin{array}{l}\text { Regions active after preparatory cue } \\
\text { Cinqulate sulcus }\end{array}$}} \\
\hline & & & \\
\hline Superior frontal gyrus (SMA) & & * & \\
\hline Frontal eye field & + & $* * *$ & \\
\hline \multicolumn{4}{|l|}{ Middle frontal gyrus } \\
\hline Inferior frontal sulcus & * & $* *$ & \\
\hline \multicolumn{4}{|l|}{ Anterior intraparietal sulcus } \\
\hline Posterior intraparietal sulcus & & * & \\
\hline \multicolumn{4}{|l|}{ Superior temporal gyrus } \\
\hline \multicolumn{4}{|l|}{ Subdivisions of visual cortex } \\
\hline Stimulus V1-V4 (upper field) & & * & * \\
\hline Stimulus V3A & & * & * \\
\hline Lower field & * & $* *$ & \\
\hline Central field & $* *$ & & \\
\hline
\end{tabular}

The above table lists the results of a series of three-way regional ANOVAs [cued location, upcoming target contrast, time (six timepoints)] on the time course of the preparatory, prestimulus BOLD response. SMA, Supplementary motor area.

${ }^{*} p=0.05 ;{ }^{* *} p=0.01 ;{ }^{* * *} p=0.001 ;{ }^{+} p=0.057$ (marginal significance).

cantly greater for leftward than rightward targets. Importantly, during high-contrast blocks, the degree of suppression of preparatory activity in regions representing the central visual field did not predict performance. Therefore, suppression of noise in unattended locations only facilitated performance when the target was difficult to detect.

\section{Discussion}

This study describes how preparatory BOLD modulations depend on the computational demands of the upcoming task. Preparing to judge the orientation of a low-contrast stimulus, relative to a high-contrast stimulus, increased activity in the FEF and in the IFS. The magnitude of these activity increases did not depend on the locus of attention, as the BOLD signal increased equally in ipsilateral and contralateral FEF and IFS. Within visual cortex, preparing for the low versus high-contrast stimulus was associated with decreased activity in regions coding unattended locations, with the strongest decreases surrounding the target location, but did not affect activity in the region coding the attended location. These effects of task cannot be explained by differences in the spatial distribution of stimuli, task difficulty, or the facilitation of task performance from carefully focused attention, because these factors were matched across low and highcontrast conditions.

\section{Task-specific suppression of unattended locations in visual cortex}

Perception of targets in high and low-contrast blocks was limited by different factors. On high-contrast trials, targets were easily visible against the background, and perception was limited by the small differences in orientation between potential targets. The challenge for the nervous system was to derive a highly accurate representation of orientation at the target location, but there was little interference from unattended locations in the immediate background. In low-contrast trials, the difference in orientation between potential targets was large, and perception was limited by the ability to detect the low-contrast target against the background. Here, the challenge for the nervous system was to sample information exclusively from the target location, because noise from the empty background could be confused with the lowcontrast target (so-called "decision uncertainty").

Many investigators have demonstrated that preparatory cues that reduce spatial uncertainty significantly improve target detection or reaction time (Posner et al., 1980; Bashinski and Bachrach, 1984; Hawkins et al., 1990; Henderson, 1991; Muller and Humphreys, 1991; Shiu and Pashler, 1994; Solomon et al., 1997; Carrasco et al., 2000; Dosher and Lu, 2000; Carrasco, 2006). The present study suggests that this effect results, at least in part, from suppression of noise in early visual areas corresponding to nearby locations, which creates a steep gradient of activity around the target location. Recent evidence suggests that decreases in BOLD activity in early visual cortex are associated with decreased neural activity (Shmuel et al., 2006). The current study suggests that a likely function of these widely reported decreases in BOLD activity for unattended locations (Tootell et al., 1998; Somers et al., 1999; Smith et al., 2000; Muller and Kleinschmidt, 2004; Silver et al., 2007) is to "mark" the location of an upcoming stimulus when it will be difficult to distinguish from the background.

It is striking that differential suppression of unattended locations was demonstrated although the stimuli in the two tasks had the same spatial distribution. Suppression of unattended locations, however, may also facilitate performance in situations in which the spatial distribution is quite different, such as when a suprathreshold target is closely surrounded by irrelevant distractors. Serences et al. (2004) measured preparatory BOLD signals in a task requiring subjects to simultaneously attend to two locations at diagonally opposite corners of a visual display, in anticipation of digit stimuli. Greater preparatory activity was reported in the portions of retinotopic visual cortex corresponding to the attended locations when subjects expected the upcoming stimuli to be surrounded by letter distractors, compared with when subjects did not expect any distractors. Serences et al. (2004) attributed the increase in the signal at the attended locations to the need to filter out the distractors. However, because the BOLD activity at distracter locations was not reported, it was unclear whether boosting the signal at the attended location potentially lessened the interfering effects of distracters by actually decreasing the signal from the distracter locations. Boosting the signal at the attended location, in the absence of any signal change at the distractor locations, could also have aided the filtering of distractors by better differentiating the target location from the distractor locations. The current work indicates that suppression of noise from nonattended locations can be mediated by a decrease in the BOLD signal directly at those locations. It is unknown whether similar task-specific preparatory decreases at unattended locations are observed when a clearly suprathreshold target stimulus is surrounded by distractors.

Results from Sapir et al. (2005) suggest that suppression of noise from the target location may also be mediated by a decrease in BOLD activity. Sapir et al. (2005) cued subjects to attend to a peripheral location in anticipation of motion stimuli embedded in external noise. Lower preparatory activity in V3A, among other regions, predicted accuracy on the upcoming motion discrimination task. This decreased activity in V3A may have reflected the same noise suppression mechanism that operated at 
A. Preparatory Activity Following Leftward Cues

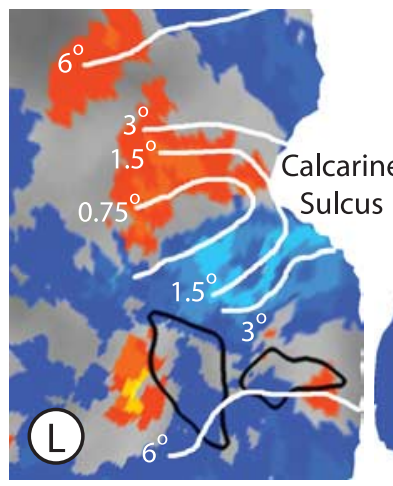

$-0.4 \%$ BOLD Change 0.4

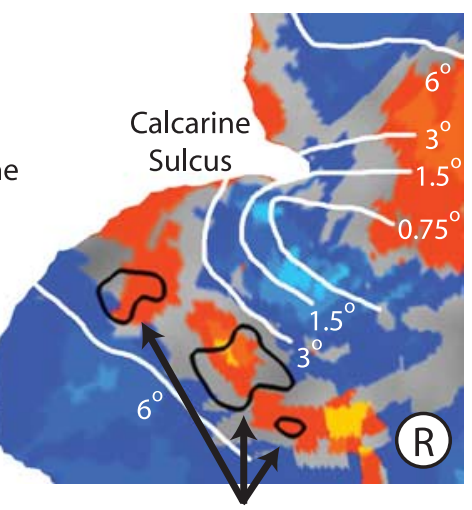

Represents Left Target Location

C. Low minus High Contrast (Leftward Cues)

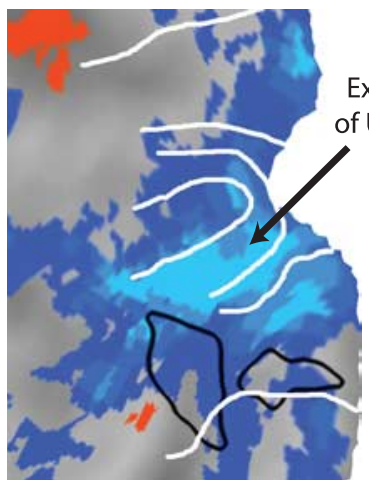

$-0.4 \%$ BOLD Change
Low $>$ High

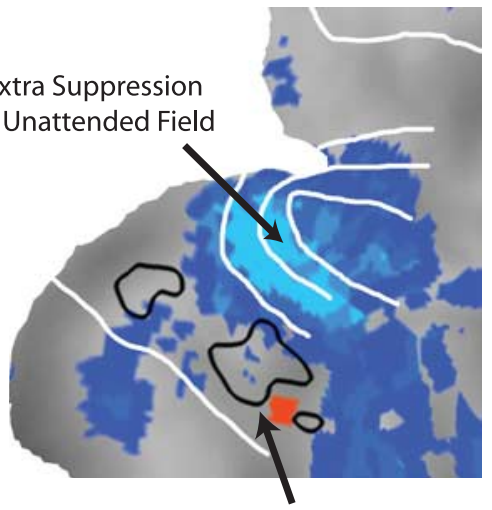

0.4 0.4 gh

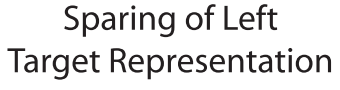

\section{B. Preparatory Activity Following Rightward Cues}

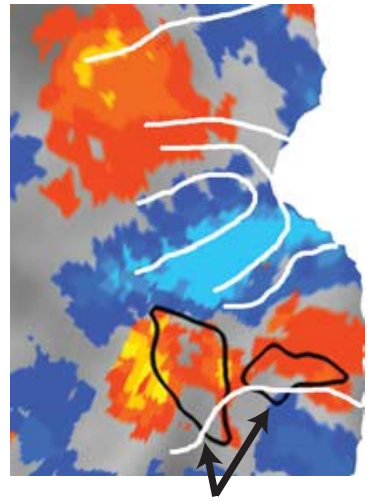

Represents Right Target Location

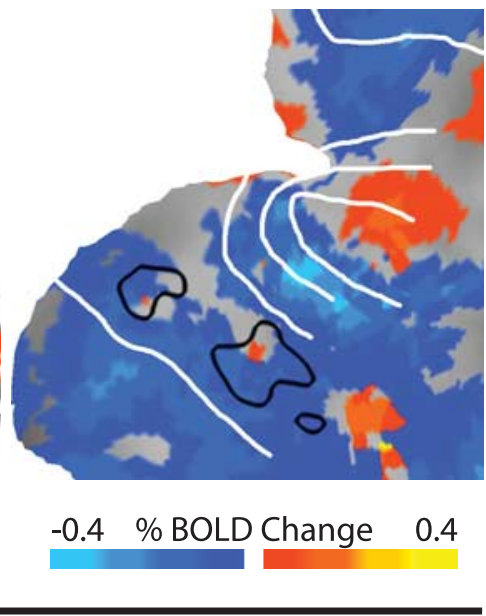

D. Low minus High Contrast (Rightward Cues)

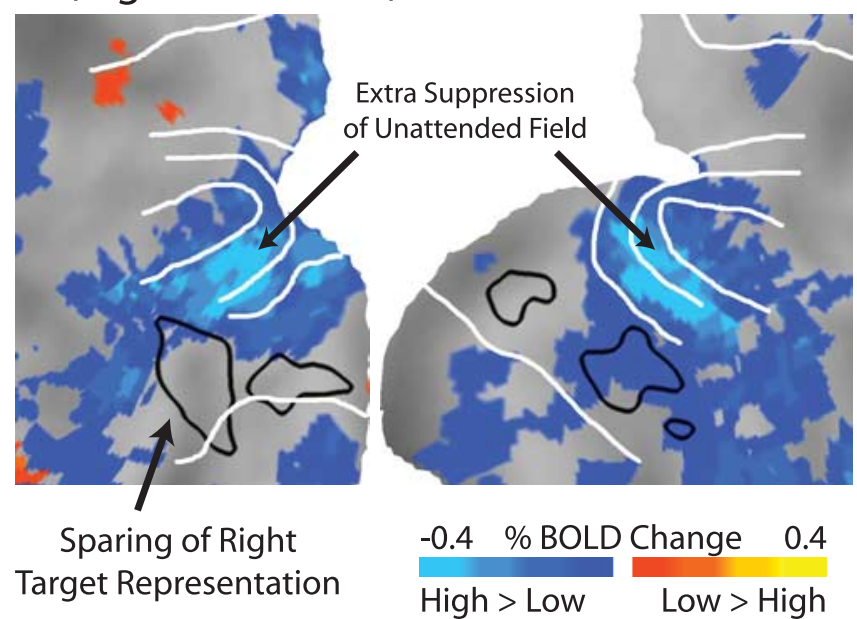

Figure 3. Preparatory activity in portions of visual cortex representing unattended locations is more suppressed in low-contrast versus high-contrast blocks. $\boldsymbol{A}, \boldsymbol{B}$, Mean BOLD activity, independent of target contrast, after auditory cues indicating the subjects should covertly attend to the upper left or upper right in preparation for visual targets. Black outlines indicate the portions of early visual cortex (V1-V4) corresponding to the target locations, as determined from independent localizer scans. $\boldsymbol{C}, \boldsymbol{D}$, Difference in preparatory activity in visual cortex in low-contrast versus high-contrast blocks, after leftward $(\boldsymbol{C})$ or rightward $(\boldsymbol{D})$ cues. Note that preparatory activity in most of visual cortex is lower in low-contrast blocks versus high-contrast blocks, suggesting extra suppression of the unattended field in low-contrast blocks. This extra suppression mostly spares the portion of cortex representing the attended location. Data maps are summed over the last two pretarget MR frames (frames 5 and 6), smoothed with a $5 \mathrm{~mm}$ full-width at half-maximum Gaussian kernel, averaged across subjects, and projected onto a flattened representation of posterior occipital cortex using the PALS (population-average, landmark-and surface-based) atlas (Van Essen et al., 2001). White lines are approximate iso-eccentricity lines based on an average of six subjects from a previous study (Jack et al., 2006).

unattended locations in the current study, because the major source of noise in the study by Sapir et al. (2005) was at the same location as the attended target.

Accurate perception in low but not high-contrast blocks was associated with increased suppression of preparatory activity corresponding to the central visual field, providing additional evidence that the suppression reflected the specific perceptual limitations of low-contrast trials. Although previous studies have linked increases in BOLD activity in retinotopic cortex to visual perception, either at the time of a target judgment (Ress et al., 2000) or before the judgment (Giesbrecht et al., 2006; Sylvester et al., 2007), this is the first study to our knowledge that links performance to preparatory BOLD decreases corresponding to unattended portions of the visual field. Suppression of the unattended field only predicted per- formance for leftward trials, probably because spatial attention was significantly more beneficial on trials with leftward versus rightward targets.

In addition to noise suppression, preparatory signals in lowcontrast trials may have also reflected signal enhancement, because the BOLD response at the attended location was clearly increased relative to baseline. This increase at the attended location is generally consistent with any of the proposed attentional modulations (e.g., additivity, contrast gain, response gain, activity gain) (McAdams and Maunsell, 1999; Martinez-Trujillo and Treue, 2002; Reynolds and Chelazzi, 2004; Williford and Maunsell, 2006) that could underlie signal enhancement during the subsequent target period. Because a similar preparatory increase at the attended location was observed in the high-contrast task it is unclear whether the top-down activity at the attended location 


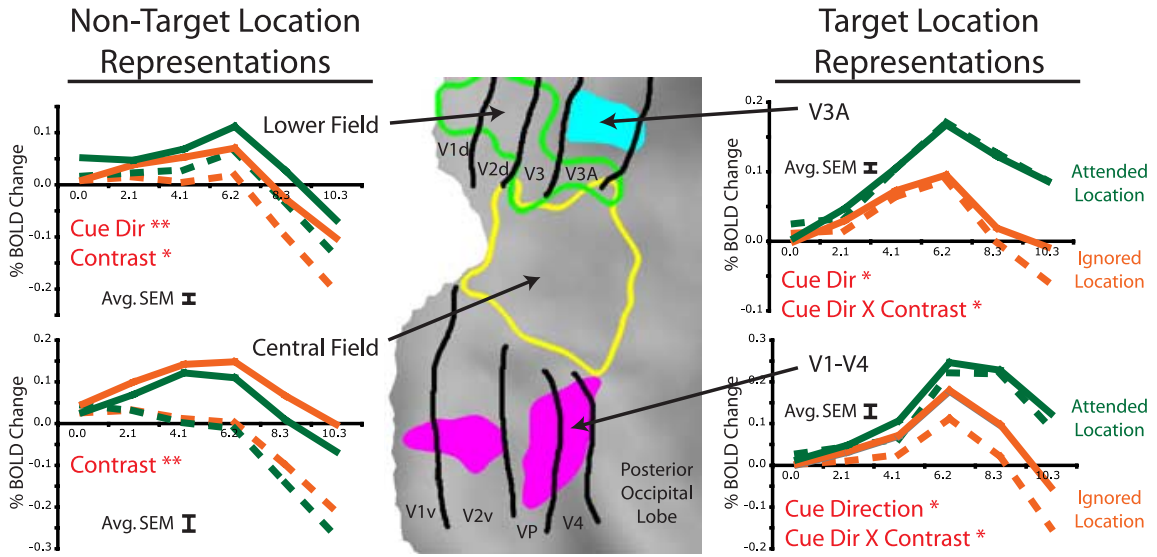

High contrast block, Contralateral cue III Low contrast block, Contralateral cue
High contrast block, Ipsilateral cue

Figure 4. Activity in regions derived from independent localizer scans confirms that BOLD activity for unattended but not attended locations is more suppressed during low versus high-contrast blocks. Activity in portions of the visual cortex representing locations that were always unattended (left panels) is always more suppressed during low-contrast relative to high-contrast blocks. Activity in portions of visual cortex corresponding to target locations (right panels) depends on which target was attended, driving a significant interaction between cue direction and contrast. When attention is directed toward the location not represented by the region (orange lines), activity is more suppressed during low-contrast blocks relative to high-contrast blocks, as is the case for all unattended locations. When attention is directed toward the location represented by the region (green lines), activity is not affected in contrast of the upcoming target. Red captions indicate significant effects from an ANOVA over the preparatory time course using subjects as a repeated measure (cue direction $\times$ contrast $\times$ time; ${ }^{*} p<0.05 ;{ }^{* *} p<0.01$ ). Regions were defined by independent localizer scans and are projected onto a flattened representation of posterior occipital cortex using the PALS (population-average, landmark- and surface-based) atlas (Van Essen et al., 2001). Black lines are approximate borders between retinotopic visual areas based on a standard atlas (Van Essen, 2002). The error bar on each graph indicates the average SEM averaged across conditions and time points.

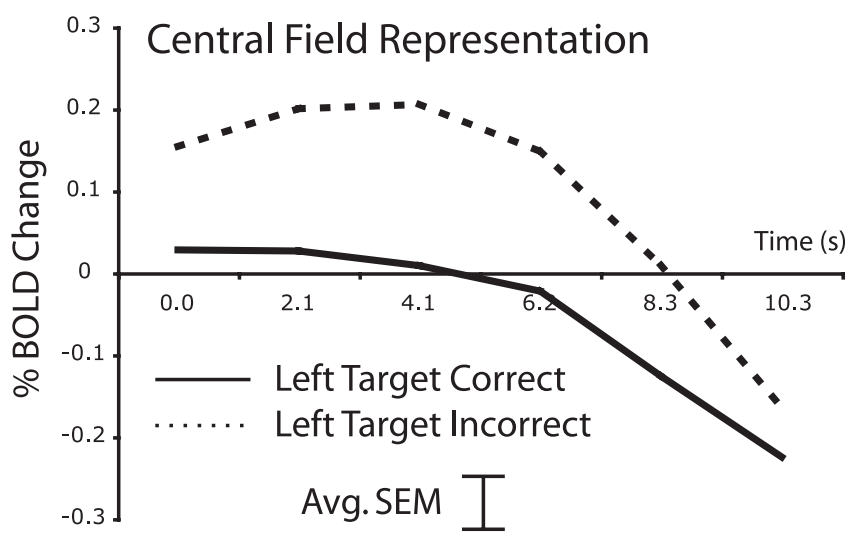

Figure 5. Greater suppression of preparatory activity in the visual cortex corresponding to the unattended central visual field predicts accurate performance for low-contrast trials with leftward targets. The error bar indicates the average SEM averaged across conditions and time points.

reflected a "signal enhancement" mechanism common to both tasks or task-specific mechanisms.

\section{Separate signals in frontal lobe for task control and spatial attention}

In addition to the extra suppression of unattended regions of retinotopic cortex, low-contrast blocks were associated with increased preparatory activity in FEF and in posterior IFS. Preparatory activity in other regions typically implicated in attentional control, such as the IPS, did not modulate with the perceptual demands of the upcoming task but only with the cued location. Although the current study did not provide evidence for a direc- tion of influence between occipital cortex and FEF or IFS, one possibility is that taskspecific frontal activity drove task-specific visual cortex activity.

Recent evidence suggests that increases in preparatory activity in FEF, but not IPS, may be causally related to suppression of the central visual field. Ruff et al. (2006, 2007) used transcranial magnetic stimulation (TMS) to stimulate either the FEF or IPS while measuring BOLD modulations in early visual cortex. In the absence of any visual input, as in the preparatory interval of the current study, stimulation of FEF (Ruff et al., 2006) but not IPS (Ruff et al., 2007) decreased activity in portions of early visual cortex (V1-V4) corresponding to the central visual field, similar to the decreases observed in the present work. Ruff et al. $(2006,2007)$ also noted increases in BOLD activity in visual cortex for all peripheral locations, whereas the current work demonstrated decreases in activity for unattended peripheral locations (i.e., the lower field) at the same eccentricity as the attended location. More selective stimulation of human FEF might produce decreases in visual cortex at selected peripheral locations.

Other studies support the hypothesis that during the low-contrast blocks of the present experiment, increased preparatory signals in FEF produced an additional suppression of activity in central retinotopic regions. Moore and Armstrong (2003) showed that microstimulation of FEF neurons decreased evoked V4 responses to stimuli in unattended portions of the visual field, perhaps indicating a similar effect on the target-evoked rather than preparatory signal. Finally, lesions of the frontal lobe result in increased excitability of primary sensory regions to distracting information, suggesting that frontal cortex may be involved in suppressing distracting information (Knight et al., 1999).

An important question is how FEF and IFS implement the spatially specific extra suppression of unattended locations in visual cortex on low-contrast trials. Any mechanism must be consistent with the observation that task signals in these higherorder regions did not depend on the locus of attention, i.e., the threshold detection task (compared with the fine discrimination task) increased signals equally in FEF and IPS regions that were ipsilateral and contralateral to the cued location. One possibility is that, during the low-contrast trials, activity only increased in the specific portions of FEF and IFS representing unattended locations. Because most of the visual field was unattended in both hemifields (e.g., all of the contralateral hemifield was unattended except for the relatively small target location), suppression of both hemifield representations in visual cortex may have involved ipsilateral and contralateral IFS and FEF to a nearly equal degree, i.e., the difference between ipsilateral and contralateral BOLD signals would not have measurably reflected the very small difference in unattended area between the two hemifields. Recent evidence from the macaque suggests that top-down projections from FEF may have the spatial specificity to support this model: microstimulation of FEF can alter the visually evoked response of V4 single units such that the response is increased only when the 
stimulus is placed within a particular portion of the V4 receptive field (Armstrong et al., 2006).

An alternative explanation is that suppression of unattended locations is mediated by a generalized activity increase across portions of the FEF and IFS representing all locations and the signal does not become spatially specific until it is relayed to visual cortex. The suppression could become spatially specific because of the manner in which cells in visual cortex combine the independent top-down signals related to the locus of attention and the need for extra suppression of the unattended visual field.

\section{Conclusions}

We described how preparatory modulations related to spatial attention are tailored to the specific perceptual demands of the upcoming task. When human subjects prepare to perceive visual objects that will be difficult to distinguish from the immediate background, our data, together with other results from neuroimaging, TMS, and single-unit studies, suggest that regions in the frontal lobe send top-down signals to visual cortex that mark the location of the upcoming stimulus. This marking is instantiated by suppressing activity in portions of visual cortex corresponding to unattended locations, while leaving activity for the attended location unchanged. The suppression causes a steep gradient in activity between portions of visual cortex corresponding to attended versus unattended locations, and may ensure that downstream brain regions are selectively modulated by information from the attended location.

\section{References}

Armstrong KM, Fitzgerald JK, Moore T (2006) Changes in visual receptive fields with microstimulation of frontal cortex. Neuron 50:791-798.

Bashinski HS, Bachrach VR (1984) Enhancement of perceptual sensitivity as the result of selectively attending to spatial locations. Percept Psychophys 28:241-248.

Brainard DH (1997) The psychophysics toolbox. Spat Vis 10:433-436.

Carrasco M (2006) Covert attention increases contrast sensitivity: Psychophysical, neurophysiological and neuroimaging studies. Prog Brain Res 154:33-70.

Carrasco M, Penpeci-Talgar C, Eckstein M (2000) Spatial covert attention increases contrast sensitivity across the CSF: support for signal enhancement. Vision Res 40:1203-1215.

Corbetta M, Shulman GL (2002) Control of goal-directed and stimulusdriven attention in the brain. Nat Rev Neurosci 3:201-215.

Dosher BA, Lu ZL (2000) Mechanisms of perceptual attention in precuing of location. Vision Res 10-12:1269-1292.

Eriksen CW, Hoffman JE (1974) Selective attention: noise suppression or signal enhancement? Bull Psychon Soc 4:587-589.

Giesbrecht B, Weissman DH, Woldorff MG, Mangun GR (2006) Pre-target activity in visual cortex predicts behavioral performance on spatial and feature attention tasks. Brain Res 1080:63-72.

Hawkins HL, Hillyard SA, Luck SJ, Mouloua M, Downing CJ, Woodward DP (1990) Visual attention modulates signal detectability. J Exp Psychol Hum Percept Perform 16:802-811.

Henderson JM (1991) Stimulus discrimination following covert attentional orienting to an exogenous cue. J Exp Psychol Hum Percept Perform 17:91-106.

Hopfinger JB, Buonocore MH, Mangun GR (2000) The neural mechanisms of top-down attentional control. Nat Neurosci 3:284-291.

Jack AI, Shulman GL, Snyder AZ, McAvoy M, Corbetta M (2006) Separate modulations of human V1 associated with spatial attention and task structure. Neuron 51:135-147.

Kastner S, Ungerleider LG (2000) Mechanisms of visual attention in the human cortex. Annu Rev Neurosci 23:315-341.

Kastner S, Pinsk MA, De Weerd P, Desimone R, Ungerleider LG (1999) Increased activity in human visual cortex during directed attention in the absence of visual stimulation. Neuron 22:751-761.

Knight RT, Staines WR, Swick D, Chao LL (1999) Prefrontal cortex regu- lates inhibition and excitation in distributed neural networks. Acta Psychol (Amst) 101:159-178.

Martinez-Trujillo J, Treue S (2002) Attentional modulation strength in cortical area MT depends on stimulus contrast. Neuron 35:365-370.

McAdams CJ, Maunsell JH (1999) Effects of attention on orientationtuning functions of single neurons in macaque cortical area V4. J Neurosci 19:431-441.

McMains SA, Fehd HM, Emmanouil TA, Kastner S (2007) Mechanisms of feature and space-based attention: response modulation and baseline increases. J Neurophysiol 98:2110-2121.

Moore T, Armstrong KM (2003) Selective gating of visual signals by microstimulation of frontal cortex. Nature 421:370-373.

Muller HJ, Humphreys GW (1991) Luminance-increment detection: capacity-limited or not? J Exp Psychol Hum Percept Perform 17:107-124.

Muller NG, Kleinschmidt A (2004) The attentional "spotlight's" penumbra: center-surround modulation in striate cortex. NeuroReport 15:977-980.

Muller NG, Bartelt OA, Donner TH, Villringer A, Brandt SA (2003) A physiological correlate of the "zoom lens" of visual attention. J Neurosci 23:3561-3565.

Pelli DG (1997) The VideoToolbox software for visual psychophysics: transforming numbers into movies. Spat Vis 10:437-442.

Posner MI, Snyder CR, Davidson BJ (1980) Attention and the detection of signals. J Exp Psychol 109:160-174.

Ress D, Backus BT, Heeger DJ (2000) Activity in primary visual cortex predicts performance in a visual detection task. Nat Neurosci 3:940-945.

Reynolds JH, Chelazzi L (2004) Attentional modulation of visual processing. Annu Rev Neurosci 27:611-647.

Ruff CC, Blankenburg F, Bjoertomt O, Bestmann S, Freeman E, Haynes JD, Rees G, Josephs O, Deichmann R, Driver J (2006) Concurrent TMSfMRI and psychophysics reveal frontal influences on human retinotopic visual cortex. Curr Biol 16:1479-1488.

Ruff CC, Bestmann S, Blankenburg F, Bjoertomt O, Josephs O, Weiskopf N, Deichmann R, Driver J (2007) Distinct causal influences of parietal versus frontal areas on human visual cortex: evidence from concurrent TMS fMRI. Cereb Cortex 18:817-827.

Sapir A, d'Avossa G, McAvoy M, Shulman GL, Corbetta M (2005) Brain signals for spatial attention predict performance in a motion discrimination task. Proc Natl Acad Sci USA 102:17810-17815.

Serences JT, Yantis S, Culberson A, Awh E (2004) Preparatory activity in visual cortex indexes distractor suppression during covert spatial orienting. J Neurophysiol 92:3538-3545.

Shiu LP, Pashler H (1994) Negligible effect of spatial precuing on identification of single digits. J Exp Psychol Hum Percept Perform 35:337-343.

Shmuel A, Augath M, Oeltermann A, Logothetis NK (2006) Negative functional MRI response correlates with decreases in neuronal activity in monkey visual area V1. Nat Neurosci 9:569-577.

Silver MA, Ress D, Heeger DJ (2007) Neural correlates of sustained spatial attention in human early visual cortex. J Neurophysiol 97:229-237.

Smith AT, Singh KD, Greenlee MW (2000) Attentional suppression of activity in the human visual cortex. NeuroReport 11:271-277.

Solomon JA, Lavie N, Morgan MJ (1997) Contrast discrimination function: spatial cuing effects. J Opt Soc Am A Opt Image Sci Vis 14:2443-2448.

Somers DC, Dale AM, Seiffert AE, Tootell RB (1999) Functional MRI reveals spatially specific attentional modulation in human primary visual cortex. Proc Natl Acad Sci USA 96:1663-1668.

Sylvester CM, Shulman GL, Jack AI, Corbetta M (2007) Asymmetry of anticipatory activity in visual cortex predicts the locus of attention and perception. J Neurosci 27:14424-14433.

Talairach J, Tournoux P (1988) Co-planar stereotaxic atlas of the human brain. New York: Thieme Medical.

Tootell RB, Hadjikhani N, Hall EK, Marrett S, Vanduffel W, Vaughan JT, Dale AM (1998) The retinotopy of visual spatial attention. Neuron 21:1409-1422.

Van Essen D (2002) Windows on the brain: the emerging role of atlases and databases in neuroscience. Curr Opin Neurobiol 12:574-579.

Van Essen DC, Drury HA, Dickson J, Harwell J, Hanlon D, Anderson CH (2001) An integrated software suite for surface-based analyses of cerebral cortex. J Am Med Inform Assoc 8:443-459.

Williford T, Maunsell JH (2006) Effects of spatial attention on contrast response functions in macaque area V4. J Neurophysiol 96:40-54. 